\title{
Rapid Communications
}

Rapid Communications are intended for the accelerated publication of important new results and are therefore given priority treatment both in the editorial office and in production. A Rapid Communication in Physical Review B should be no longer than 4 printed pages and must be accompanied by an abstract. Page proofs are sent to authors.

\section{Skyrmion excitation in the infinite- $U$ Hubbard model}

\author{
T. K. Ng \\ AT\&T Bell Laboratories, Murray Hill, New Jersey 07974
}

(Received 22 October 1990)

\begin{abstract}
The energy of single Skyrmion excitation above the Nagaoka state is being studied numerically in the infinite- $U$ Hubbard model in the limit of small concentration of holes. We find that the energy of a Skyrmion is low compared with energy of other spin excitations being studied, suggesting the Skyrmions may be important in describing the behavior of the infinite- $U$ Hubbard model near half filling.
\end{abstract}

With the recent uprise of interest in the single-band Hubbard model, the question of what is the ground state of the infinite- $U$ Hubbard model close to half filling has received renewed attention. In the limit of a single hole in an otherwise half-filled band, saturated ferromagnetism (the "Nagaoka" state) ${ }^{1}$ is known to occur. On the other hand, for large hole concentrations, the ground state is believed to be paramagnetic. ${ }^{2}$ The question of interest is how are these two limits connected, or whether the Nagaoka state remains stable at all once a finite concentration of holes is introduced in the system.

The problem is usually tackled in the following way. Consider the system with a finite concentration of holes $\delta$ and is in the Nagaoka (saturated ferromagnetism) state. Now consider a single spin-flip excitation. If the energy of this excitation is negative, the Nagaoka state is unstable. This line of approach has been carried out by exact diagonalization of a few holes in finite systems $s^{3,4}$ and also by constructing approximate spin-flip excitation wave functions where the overturned spin either propagates coherently ${ }^{5,6}$ (Gutzwiller-type wave function) or is localized. $^{7,8}$

In this paper we shall consider an alternative kind of spin-flip excitation where the overturned spins form a localized Skyrmion. We shall study the energy of a Skyrmion at a small concentration of holes $\delta(\delta \rightarrow 0)$ and compare the energy with energies of other approximate spinflip excitations.

The Hamiltonian for the infinite- $U$ Hubbard model can be written in the slave fermion Schwinger boson representation as ${ }^{9}$

$$
H=-\sum_{\langle i j\rangle_{\sigma}} t_{i j} \bar{Z}_{i \sigma} Z_{j \sigma} \psi_{j}^{\dagger} \psi_{i}
$$

with constraint $\Sigma_{\sigma} \bar{Z}_{i \sigma} Z_{i \sigma}+\psi_{i}^{\dagger} \psi_{i}=1$, where $Z_{i \sigma}$ is a spin$\sigma$ Schwinger boson operator and $\psi_{i}$ is a spinless fermion operator for holes.
In the small concentration of hole $\delta \rightarrow 0$ limit, one can consider the continuum limit of Eq. (1), ${ }^{9}$

$$
\begin{gathered}
H \rightarrow \frac{-1}{2 M} \int\left[\psi^{\dagger}\left(\partial_{\mu}-i A_{\mu}\right)^{2} \psi-\gamma \psi^{\dagger} \psi\left|\left(\partial_{\mu}-i \beta_{\mu}\right) Z\right|^{2}\right. \\
\left.-\psi^{\dagger} \psi\left(A_{\mu}-\beta_{\mu}\right)^{2}\right] d^{2} x
\end{gathered}
$$

where $\left|\left(\partial_{\mu}-i \beta_{\mu}\right) Z\right|^{2}=(\nabla \mathbf{S})^{2}$ represents the energy coming from the twist of the magnetization $S$ (i.e., spin waves), $A_{\mu} \sim i \bar{Z} \partial_{\mu} Z, \beta_{\mu} \sim i \psi^{\dagger} \partial_{\mu} \psi /\left|\psi^{\dagger} \psi\right|$ are the gauge fields associated with the double spin twists ${ }^{10}$ and hole motions, respectively. $\gamma>0$ describes a hole-spin wave repulsion with strength depending on the underlying lattice structure, for usual lattices $\gamma \sim 1$. In deriving the above Hamiltonian, we have neglected terms of order $\delta^{2}$, thus Eq. (2) is, strictly speaking, applicable only in the $\delta \rightarrow 0$ limit.

The Nagaoka state is obtained in the above Hamiltonian by setting $Z(x)=$ const everywhere and with $A_{\mu}=\beta_{\mu}$ $\equiv 0$. The one Skyrmion state can be obtained in a similar way by setting ${ }^{10}$

$$
Z(x, y)=W /|W|,
$$

where

$$
W=\left(\begin{array}{l}
x+i y-a \\
x+i y+a
\end{array}\right)
$$

and $A_{\mu}=\beta_{\mu}=i \bar{Z} \partial_{\mu} Z$, describing a Skyrmion of size $\lambda=a$ located at the origin. Notice that unlike the Nagaoka state, this Skyrmion state is not an exact eigenstate of the system, but should be regarded as a mean-field approximation for the "real" Skyrmion excitation. The energy of this "mean-field" Skyrmion state can be computed by solving the Schrödinger equation for holes moving in the effective $e-m$ potential generated by the Skyrmion-spindistribution $W /|W|$. In the gauge $A_{r}=0$, we obtain the 
Schrödinger equation

$$
\begin{array}{r}
\frac{-1}{2 M}\left(\frac{\partial^{2}}{\partial r^{2}}+\frac{1}{r} \frac{\partial}{\partial r}-\frac{1}{r^{2}}\left(m-r A_{\phi}\right)^{2}-\gamma B(r)\right) U_{m}(r) \\
=E U_{m}(r)
\end{array}
$$

where

$$
A_{\phi}=\frac{r}{r^{2}+a^{2}}, B(r)=\frac{2 \lambda^{2}}{\left(r^{2}+\lambda^{2}\right)^{2}},
$$

and $m$ equal to an integer is the (canonical) angular momentum of the wave function.

The equation can be simplified by writing $k^{2}=2 M E$, $k r=x$, and $\lambda=k a$, resulting in

$$
\begin{aligned}
{\left[\frac{d^{2}}{d x^{2}}+\frac{1}{x} \frac{d}{d x}+1-\frac{1}{x^{2}}\right.} & \left(m-\frac{x^{2}}{x^{2}+\lambda^{2}}\right)^{2} \\
& \left.-\frac{2 \gamma \lambda^{2}}{\left(x^{2}+\lambda^{2}\right)^{2}}\right] U_{m \lambda}(x)=0,
\end{aligned}
$$

which describes scattering of two-dimensional plane waves $U_{m \lambda}(x)$ by an angular-momentum-dependent central potential

$$
V_{m \lambda}(x)=\frac{2 m \lambda^{2}}{x^{2}\left(x^{2}+\lambda^{2}\right)}-\frac{\lambda^{4}}{x^{2}\left(x^{2}+\lambda^{2}\right)^{2}}+\frac{2(\gamma-1) \lambda^{2}}{\left(x^{2}+\lambda^{2}\right)^{2}} .
$$

(Notice that the kinetic angular momentum of the incoming plane wave is $m-1$ but not $m$, because the Skyrmion carries a total magnetic flux of one flux quantum. ${ }^{10}$ ) The energy of the Skyrmion can be computed by first determining the scattering phase shift $\Delta$ of incoming waves due to $V_{m \lambda}(x)$ and then using the Fumi's theorem ${ }^{11}$

$$
\begin{aligned}
E_{s} & =-\int_{0}^{E_{F}} d E \sum_{m} \frac{\Delta_{m}(E)}{\pi} \\
& =\frac{-2 E_{F}}{\lambda_{F}^{2}} \int_{0}^{\lambda_{F}} \lambda d \lambda \sum_{m} \frac{\Delta_{m}(\lambda)}{\pi},
\end{aligned}
$$

where $\lambda_{F}=k_{F} a$. $k_{F}=(4 \pi \delta)^{1 / 2}$ is the Fermi momentum of holes and $E_{F}$ the Fermi energy. Notice that the size of Skyrmion $a$ comes in only as the ratio $\lambda_{F}=k_{F} a$.

We have solved Eq. (5) numerically for different values of $m$ and $\lambda$ and the phase shift $\Delta_{m}(\lambda)$ is determined by fitting at large $x$ the function $U_{m \lambda}^{\prime}(x) / U_{m \lambda}(x)$ to $\tilde{U}_{m \lambda}^{\prime}(x) / \tilde{U}_{m \lambda}(x)$, where

$\tilde{U}_{m \lambda}(x)=\left[\cos \Delta_{m}(\lambda)\right] J_{m-1}(x)-\left[\sin \Delta_{m}(\lambda)\right] N_{m-1}(x)$,

$J_{m}$ and $N_{m}$ are the Bessel and Neumann functions, respectively. Notice that $U_{m \lambda}(x) \rightarrow x^{|m|}$ as $x / \lambda \rightarrow 0$ (Ref. 12) because of the singular $V_{m \lambda}(x)$, reflecting the change in angular momentum because of the Skyrmion magnetic field $A_{\phi}$ as $x$ increases from zero to infinity.

In Fig. 1 we plot the energy of a Skyrmion in units of $E_{F}=k_{F}^{2} / 2 M$ as a function of the size parameter $\lambda_{F}=k_{F} a$ for $\gamma=0$ and 1. As is evident from the figure, in the $\gamma=1$ case, the energy of the Skyrmion is dominated by the hole-spin wave repulsion term, at least for $\lambda_{F} \gtrsim 0.3$. The effect of this term can be understood quite easily. In the

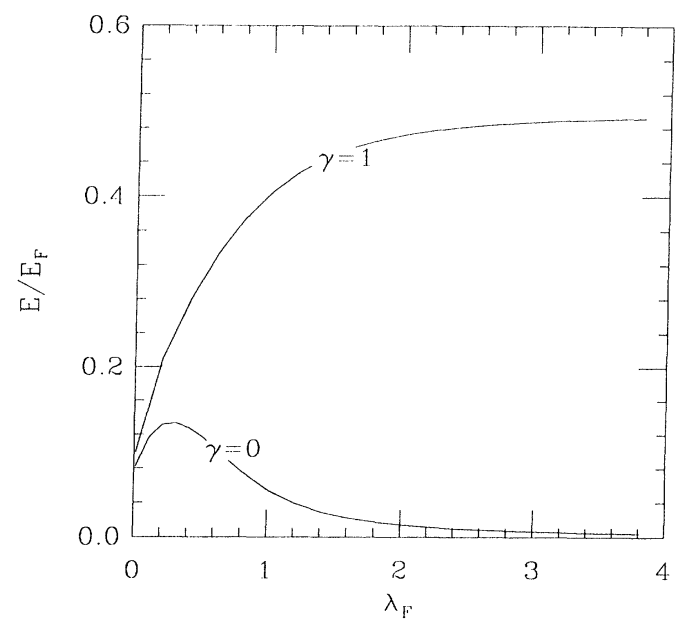

FIG. 1. Energy of a Skyrmion in units of $E_{F}=k_{F}^{2} / 2 M$ as a function of the size parameter $\lambda_{F}$ for two values of $\gamma$.

$\lambda_{F} \rightarrow 0$ limit $B(\mathbf{r}) \rightarrow 2 \pi \delta^{(2)}(\mathbf{r})$, and its effect vanishes logarithmically (as $\lambda_{F} \rightarrow 0$ ). In the limit $\lambda_{F} \rightarrow \infty$, it becomes a constant potential with total strength $s=\pi / M$ which results in the constant energy contribution $E=\delta s=E_{F} / 2$. The effect of the $A_{\phi}$ term also can be easily understood. In the limit $\lambda_{F} \rightarrow 0$, it describes a flux tube with total flux of one flux quantum and its effect vanishes logarithmically. In the other limit $\lambda_{F} \rightarrow \infty$, it becomes a uniform magnetic field of vanishing strength and its effect vanishes. Notice that for both values of $\gamma$, the energy $E$ should vanish logarithmically as $\lambda_{F} \rightarrow 0$, which is not observable in the scale given in Fig. 1. Notice also that the energy of the Skyrmion is always positive, indicating that the Nagaoka state is stable toward formation of Skyrmion, at least in the small $\delta$ limit. This result is quite nontrivial at least in the $\gamma=0$ case, since $V_{m \lambda}$ is attractive for $m \leq 0$ and repulsive for $m \geq 1$.

Next, we shall consider the case when the underlying lattice is a square lattice with nearest-neighbor hopping $t$. In this case, $\gamma=1$ and $M=(2 t)^{-1}$ (we take the lattice spacing $r_{0}=1$ ). We shall study the energy of fixed size Skyrmions as functions of the hole concentration $\delta$. In Fig. 2, we plot the energies of two Skyrmions with fixed sizes $a=1,3$ (solid lines) in units of $2 t$ as functions of $\delta$ for small $\delta$. The corresponding energy of a hard-core potential with radius $a=1$ is also given for comparison (dashed line). Notice that because of the underlying lattice, strictly speaking, a Skyrmion is well defined only when $a \gg 1$. Notice also that the hard-core potential with $a=1$ can be visualized as describing in the continuum limit a single localized overturned spin in the Nagaoka state in the infinite- $U$ Hubbard model, where holes cannot penetrate.

From Fig. 2, we find that the energy of a Skyrmion increases as $\delta$ increases, as is expected from our previous consideration. Also, we find that the energy of the hardcore potential is always higher than the energy of the Skyrmion with the same size $(a=1)$. The energy of the hard-core potential also increases much more rapidly than the energy of a Skyrmion as $\delta$ increases. In fact, we find 


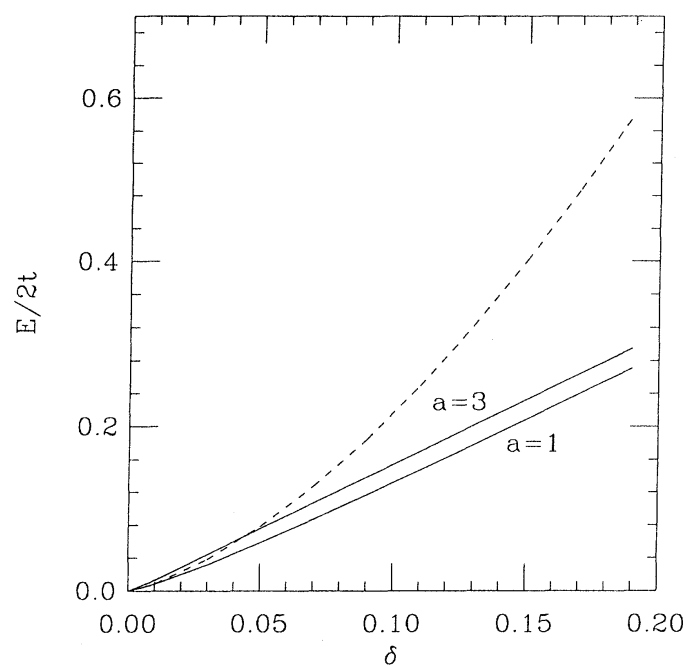

FIG. 2. Energy of Skyrmions with fixed sizes $a=1,3$ (solid lines) in units of $2 t$ as function of hole concentration $\delta$. The energy of a hard-core potential with radius $a=1$ as function of $\delta$ is also plotted for comparison (dashed line).

that the energy of the hard-core potential becomes higher than the energy of the $a=3$ Skyrmion as $\delta \gtrsim 0.04$.

The reason for this behavior is simple, the hard-core potential is a much stronger (repulsive) potential than the Skyrmion potential, especially for large incoming momentum $k$. (The hard-core potential phase shift $\Delta \sim k a$ for large $k$, while $\Delta$ is approximately constant for Skyrmion when $\lambda=k a$ is large, as is explained above.) The hardcore potential becomes "weak" only in the $\delta \rightarrow 0$ (or $k_{F} \rightarrow 0$ ) limit, when the phase shift $\Delta$ vanishes logarithmically and its energy becomes comparable with Skyrmion of similar size.

Recently, Kopp, Ruckenstein, and Schmitt-Rink ${ }^{8}$ show that the energy of a localized overturned spin in the Nagaoka state on a square lattice is lower than the energy of a overturned spin propagating coherently, over a very large range of $\delta<\delta_{\mathrm{cr}}^{\prime}$. Based on the result, they concluded that in the small $\delta$ limit, the spin-flip excitation spectrum of the Nagaoka state should have a very large incoherent part coming from reactions of the "hole" Fermi sea to the potential created by the overturned spin, although a finite quasiparticle weight still exists for a traveling "overturned" spin ${ }^{8}$ in two dimensions. Our results suggest that the energy of a small-size Skyrmion excitation is even lower and more stable, at least for not too small $\delta$, when the Skyrmion is not too small to be well defined. In the original spin language, the reason why a Skyrmion has a lower energy is that while a hole cannot penetrate through a localized overturned spin without moving it, it can penetrate through a Skyrmion more easily because the spins are twisting more smoothly. (A similar mechanism is responsible for the formation of spiral phases in the $t-J$ model, ${ }^{13,14}$ where a hole cannot propagate through a classical Néel state background but can propagate through a smoother "spiral" spin state.) Notice that this mechanism is independent of the concentration of holes and the lattice structure, thus we expect that some localized spin states with smoother spin distribution will have lower energy than a single, localized overturned spin state even in the $\delta \rightarrow 0$ limit, although we cannot study it in the continuum model. The Green's function spectrum of a "traveling" Skyrmion should be qualitatively very similar to that of a traveling overturned spin since they interact similarly with the hole Fermi sea. The main difference is that a Skyrmion has a "weaker" interaction with the Fermi sea (smaller phase shift) and thus the incoherent part of the Skyrmion spectrum should be "smaller."

To summarize, we have studied in this paper singlelocalized-Skyrmion excitations above the Nagaoka state in the continuum limit of the infinite- $U$ Hubbard model. We have constructed a mean-field Skyrmion state and studied its energy numerically. We find that for all cases we study, the energy of Skyrmion is always positive, indicating that the Nagaoka state is always stable with respect to formation of a single Skyrmion. We also find that the energy of a Skyrmion is always lower than the energy of a hard-core potential of the same radius, indicating that Skyrmion-like excitation may have lower energy than single-overturned-spin excitation in the infinite- $U$ Hubbard model over a large range of hole concentration $\delta$ when the Skyrmion is not too small to be well defined. The main drawback of our work is the use of a meanfield-type approximation where fluctuation in spin configuration when holes propagate are neglected. Quantum fluctuation of spin configuration is expected to be important in the present model because of the small spin magnitude $\left(S=\frac{1}{2}\right)$ involved. Thus it is expected that the "true" Skyrmion state will always be strongly renormalized from the mean-field theory. Moreover, because of the existence of an underlying lattice, the topological nature of the Skyrmion is not going to guarantee that Skyrmions are stable (which can be seen from the fact that a small-size Skyrmion is not well defined). The effect of quantum fluctuation on the Skyrmion state is not clear at present.

However, we expect that the energy of the "true" Skyrmion state, if stable, will still be lower than the energy of a localized, overturned spin because of the physical reason that the hole can propagate through a "smoother" spinconfiguration more easily. Notice, however, that this argument breaks down if recoil of the overturned spin is allowed in the overturned spin wave function, in which case a full analysis of the problem is required and is outside the scope of the present paper.

Another interesting problem which we have not addressed in this paper is whether a Skyrmion can be stabilized (with respect to the Nagaoka state) by forming a Skyrmion lattice where the energy of the hole is lowered by coherence effects. We shall address this question in a separate paper.

The author thanks B. Shraiman and S. Liang for helpful discussions. 
'Y. Nagaoka, Phys. Rev. 147, 392 (1966).

2J. Kanamon, Prog. Theor. Phys. 30, 275 (1973); see also D. C. Mattis, The Theory of Magnetism (Springer, Berlin, 1981).

${ }^{3}$ J. A. Riera and A. P. Young, Phys. Rev. B 40, 5285 (1989).

${ }^{4}$ Y. Fang, A. E. Ruckenstein, E. Dagotto, and S. Schmitt-Rink, Phys. Rev. B 40, 7406 (1989).

${ }^{5}$ B. S. Shastry, H. R. Krishnamurthy, and P. W. Anderson, Phys. Rev. B 41, 2375 (1990).

${ }^{6}$ A. G. Basile and V. Elser, Phys. Rev. B 41, 4842 (1990).

${ }^{7}$ P. Richmond and G. Rickayzen, J. Phys. C 2, 528 (1969).

${ }^{8}$ T. Kopp, A. E. Ruckenstein, and S. Schmitt-Rink (unpublished).
${ }^{9}$ L. B. Ioff and P. B. Wiegmann, Phys. Rev. Lett. 65, 653 (1990).

${ }^{10} \mathrm{See}$, for example, A. M. Polyakov, Gauge Fields and Strings (Harwood, Academic, Char, Switzerland, 1987).

${ }^{1}$ See, for example, C. P. Mahan, Many-Particle Physics (Plenum, New York, 1981).

${ }^{12}$ This result can be obtained by a series expansion of $U_{m \lambda}(x)$ around $x=0$.

${ }^{13}$ B. Shraiman and E. Siggia, Phys. Rev. Lett. 62, 1564 (1989).

${ }^{14}$ C. L. Kane, P. A. Lee, T. K. Ng, B. Chakraborty, and N. Read, Phys. Rev. B 41, 2653 (1990). 